\title{
Impact of polyphenols on mast cells with special emphasis on the effect of quercetin and luteolin
}

\author{
YASDANI SHAIK ${ }^{1}$, ALESSANDRO CARAFFA', GIANPAOLO RONCONI ${ }^{3}$, \\ GIANFRANCO LESSIANI ${ }^{4}$, PIO CONTI ${ }^{5}$
}

\author{
${ }^{1}$ Department of Medicine, Boston University School of Medicine, Boston, USA \\ ${ }^{2}$ Department of Pharmacology, University of Perugia, Perugia, Italy \\ ${ }^{3}$ Clinica dei Pazienti del Territorio, Policlinico Gemelli, Roma, Italy \\ ${ }^{4}$ Internal Medicine, Villa Serena Hospital, Cittŕ Sant'Angelo, Italy \\ ${ }^{5}$ Universitŕ G. d'Annunzio, Chieti-Pescara, Italy
}

\begin{abstract}
Polyphenols are ubiquitous in food and have long been recognized to possess antioxidant, antiinflammatory and anticancer activities. Mast cells (MCs) are implicated in the pathogenesis of inflammatory diseases, allergy, autoimmunity and cancer. MCs derive from hematopoietic progenitor cells, reside virtually in all vascularized tissue and are activated by crosslinking of FceRI-bound IgE (at very high affinity: $1 \times 10^{10} \mathrm{M}^{-1}$ ) with multivalent antigen. MCs in cytoplasmic granules release preformed chemical mediators, and also they can release lipid mediators and cytokines/chemokines without degranulation.

Luteolin, 3',4',5,7-tetrahydroxyflavone, is a flavonoid contained in many kinds of plants including vegetables and fruits. This anti-oxidant product inhibits interleukin (IL)-6, IL-8 and vascular endothelial growth factor (VEGF) production from tumor necrosis factor (TNF)-triggered keratinocytes, and is a candidate for use in alternative therapies in the treatment of inflammatory skin disorders.

Quercetin (3,3',4',5,7-pentahydroxyflavone) is a ubiquitous flavonoid which exhibits anti-cancer, anti-oxidative and anti-inflammatory properties and causes a reduction in the availability of nitrite that influences vascular function. Quercetin exerts physiological functions though the interaction with phosphatidylinositol-3-phosphate kinase (PI3K), mitogen-activated protein kinase (MAPK), extracellular signal regulated kinase (ERK), kinase (MEK) 1, and others, and has a negative effect on FceRI cross-linking and other activating receptors on mast cells. In this article we report for the first time the interrelationship between mast cells and polyphenols.
\end{abstract}

Key words: polyphenols, mast cells, immunity, inflammation.

(Centr Eur J Immunol 2018; 43 (4): 476-481)

At the beginning of the last century, Rusznyak and Szent-Gyorgyi reported that citrus fruits contain diverse substances other than vitamin $\mathrm{C}$, which can prevent capillary fragility. These substances were the polyphenols (flavonoids, phenolic acids, lignans, coumarins) or flavonoids contained in large quantities in fruit, vegetables, cereals, and beverages, and which may have a positive effect under conditions of stress and other neurological dysfunctions [1]. Constituents of grapes, such as quercetin, resveratrol, kaempferol, catechin, epicatechin and anthocyanins, constitute more than $70 \%$ of the grape polyphenols. Natural compounds have long been recognized to possess antiinflammatory, antioxidant, antiallergic, hepatoprotective, antithrombotic, antiviral and anticancer activities.
Flavonoids, previously called vitamin $\mathrm{P}$ or vitamin $\mathrm{C} 2$, are numerous; in fact, to date, about 800 different flavonoids have been isolated. Flavonoids are polyphenolic secondary metabolites classified into anthocyanins, flavonols, flavones, flavan-3-ols, flavanones, isoflavones, and chalcones; they are ubiquitous in food and potentiate the anti-scorbutic activity shown in a number of animal studies. Flavonoids cannot be considered vitamins, since they produce pharmacologic effects rather than nutritional ones. It is well known that polyphenols such as coumarin, curcumin, catechin, resveratrol, anthocyanidin, tannin, rutin, isoflavone, quercetin, etc. exert antioxidant properties, and these are found in red wine, chocolate, tea, pomegranate and fruit juices, where they are the greatest contributors of flavonoids in the human diet along with vegetables [2]. 


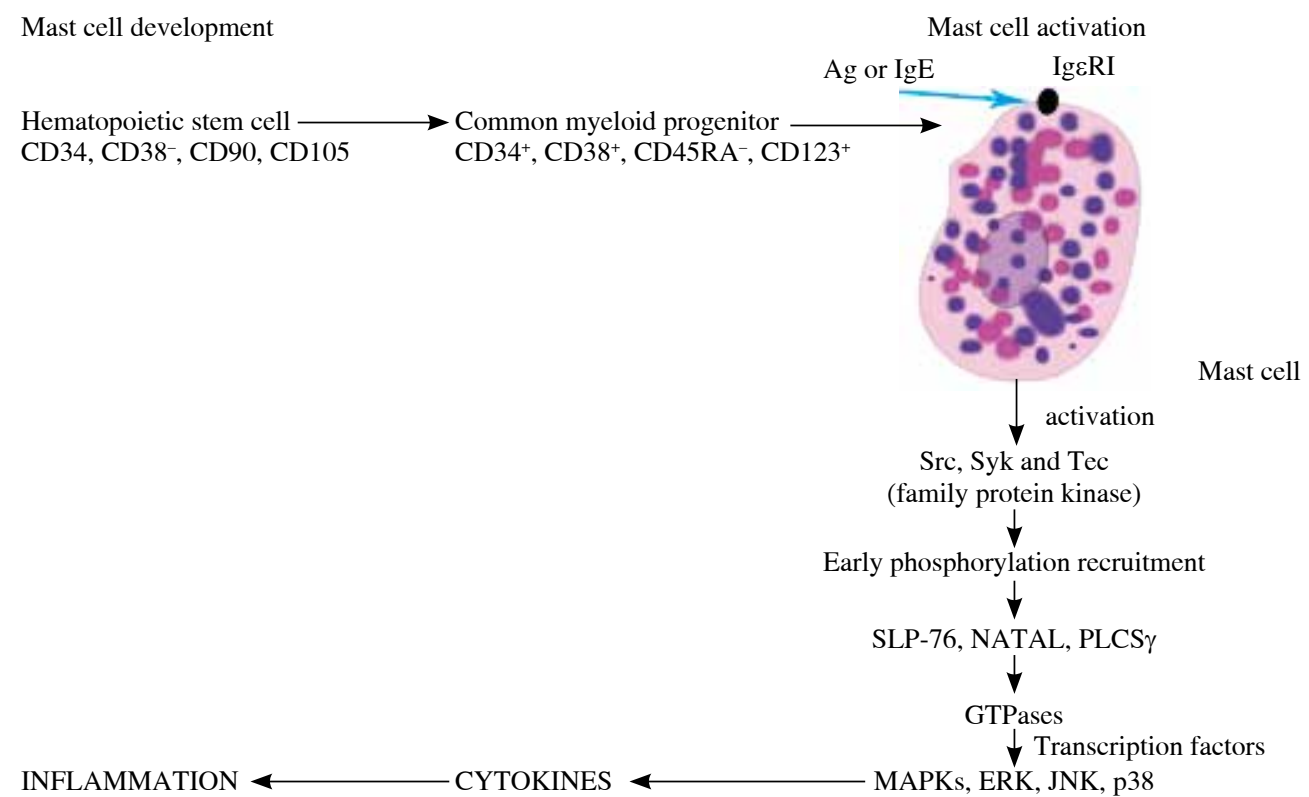

Fig. 1. Mast cell activation by IgE or antigen and cytokine provoking inflammation

The hydroxyl groups of these polyphenols enable them to form glycosidic linkages with sugars. Most polyphenols occur naturally as glycosides which are hydrolyzed and converted into their respective aglycones by lactase phlorizin hydrolases on the cell surface as well as cellular and bacterial, prior to absorption by glycosidases of the intestinal flora [3].

Found in plants, polyphenols, which are colored phenolic substances, comprise the major sources of red, blue and yellow pigments which exhibit pleiotropic biological activities such as tissue protection, reduced capillary fragility and/or permeability, antioxidant protecting effects and chelating properties such as the ability to chelate divalent metal cations (e.g., $\mathrm{Cu}^{++}, \mathrm{Fe}^{++}$). Their antioxidant functions are exerted by removing the catalysts of lipid peroxidation reactions, aldose reductase, phosphodiesterases, and o-methyltransferase [4]. However, no clinical diseases associated with polyphenol deprivation have been reported.

The large number of flavonoids leads to many different mechanisms of action and often they are tested in clinical trials [5]. They have neurological effect and therefore are used as antidepressants [6]. Some flavonoids exert an antidepressant effect by elevating glucocorticoid receptors, serotonin norepinephrine and/or brain-derived neurotrophic factor, modulating the hypothalamus-pituitary-adrenal axis [7].

It has been reported that certain natural flavonoids have anti-inflammatory activities, used for neuropsychiatric disorders [8]. Their intake is associated with decreased incidence of dementia and improvement of cognitive dysfunctions [9].
There is reason to believe that oxygen radicals are involved in the pathophysiology of inflammatory cells which generate and release reactive oxygen species. The antioxidative properties of polyphenols are considered to benefit human health, and the antioxidant flavonoid quercetin prevents oxidative stress and leads to its reduction [10]. The antioxidant polyphenols can turn off the reactive oxygen species (ROS) activity and prevent cellular tissue damage by reacting with oxidizing free radicals [11], and therefore can be used for therapies in diverse pathologies such as autoimmune diseases, inflammatory disorders and cancer [12]. However, whether polyphenols alter biological effects during their therapeutic and preventive actions remains unclear. It has been reported that polyphenols may alleviate the adverse effects of chemotherapy and radiotherapy, but may antagonize antitumor effects by reducing oxidative damage [13].

Whether polyphenols can antagonize antitumor effects of radiotherapy and chemotherapy remains unclear [12]. They may modulate gene expression influencing cellular proliferation and differentiation [14]. Moreover, it may cause the modulation of the intracellular kinase pathways p38, mitogen-activated protein kinase (MAPK), and extracellular regulated kinase (ERK). Polyphenols may downregulate inflammatory responses in LPS-activated murine peritoneal macrophages, suppressing NFKB and MAPK signaling pathways [15]

The Janus kinase activation (JAK)-STAT pathway is an important signal transduction pathway for numerous cytokines and chemokines, which binds its receptor and leads to JAK activation and phosphorylates STATs [16]. Since the JAK-STAT pathway plays a crucial role in dysregula- 
tion of the T-cell response and is involved in inflammatory and allergic diseases, it is likely that these signals are influenced by polyphenols.

Mast cells (Fig. 1) derive from a distinct precursor in the bone marrow and are multifunctional immune cells implicated in the pathogenesis of inflammatory disease allergy, autoimmunity and cancer [17]. Crosslinking of FceRI with antigen or IgE induces Lyn activation, which phosphorylates the immunoreceptor tyrosine-based activation motif (ITAM). ITAM- $\gamma$ chain recruits spleen tyrosine kinase (Syk) to the receptor. This phosphorylation activates phospholipase C gamma (PLC- $\gamma$ ), leading to the stimulation of inositol 1,4,5-triphosphate (IP3), which regulates the calcium $\left(\mathrm{Ca}^{2+}\right)$ flux and diacylglycerol (DAG), which acts on PKC activity. Therefore, in MCs, these signal pathways and others can cause immediate degranulation and/ or the late generation of arachidonic acid products, cytokines and chemokines. Mast cells can perform important beneficial roles in host defense, and they can secrete mediators without overt degranulation [18]. Human mast cells contain and release several inflammatory compounds and are essential for allergies, innate and acquired immunity, and inflammation [19]. They mediate all these processes through the release of various mediators: histamine, tryptase ( $\alpha$ and $\beta$ ), chymase, proteoglycans, prostaglandin D2, and produce leukotrienes, whose receptors are expressed on rat microglia [20]. Tryptase activates protease-activated receptors (PARs), which are G-protein-coupled receptors, and participate in allergic diseases and inflammatory reactions mediated by human mast cells [21]. Increased secretion of mast cell tryptase in certain disorders, such as bronchial asthma, may augment neurogenic inflammation [22].

Under stress, activated mast cells, which have been proposed as an immune gate to the brain, as well as environmental sensors, generate cytokines/chemokines which together with neuropeptides are involved in the development of several neuropathological processes, and are implicated in inflammation of the central nervous system [23]. The release of neuropeptides from sensory nerves causes an increase in vascular permeability, plasma extravasation and edema [24]. Mast cells are stimulated and activated by cytokines, growth factors, hormones, and bacterial and viral compounds, leading to differential release of distinct mediators without degranulation. These effects appear to involve de novo synthesis of mediators, such as vascular endothelial growth factor and interleukin (IL)-6 [25].

Interleukin 6 is a pleiotropic cytokine produced by Th2 cells or mast cell activation. IL- 6 mediates various pathophysiological processes, such as immune responses, inflammation, acute phase responses, haematopoiesis, and acute stress, through activation of the hypothalamicpituitary-adrenal axis [26]. IL-6 is important for mast cell maturation and proliferation. This cytokine has been shown to exacerbate several neurological dysfunctions induced or mediated by other cytokines [27]. Mast cells can accumulate in inflammatory tissues in response to a specific $\mathrm{C}-\mathrm{C}$ chemokine, such as regulated on activation, normal T-cell expressed and secreted (RANTES) and MCP-1. Injections of hrRANTES or monocyte chemoattractant protein-1 (MCP-1) in skin tissue provided direct in vivo evidence that RANTES has a significant effect on mast cell recruitment and histidine decarboxylase (HDC) mRNA generation [28]. HDC is an important mast cell biochemical and functional marker for the generation of histamine from histidine. Histamine is expressed by neurons involved in many brain functions [29]. The activity of the HDC enzyme is altered in various neurological diseases [30]. The flavonoid quercetin has a variety of functions including anti-allergic and neuroprotective activities, and it is known to inhibit histamine release from human basophils and murine mast cells [31]. Cloning the HDC cDNA probe into the p-Mal plasmid and using it for Northern blot hybridization, we previously showed that quercetin has an inhibitory effect on HDC mRNA, and tryptase and IL-6 release by the human mast cell line HMC-1 [27]. The suppression of HDC mRNA transcription and tryptase and IL-6 production by quercetin on mast cells may clarify in part many pathological conditions associated with several inflammatory disorders, including neurological diseases and allergies [32].

Quercetin (3,3', 4', 5,7-pentahydroxyflavone) is a ubiquitous flavonoid contained in vegetables and fruits and is one of the most widely popular flavonoids ingested in food by humans [33]. After quercetin ingestion through vegetables and/or fruits, quercetin glycosides are metabolized, absorbed, and circulated in the blood. Subsequently, after conjugation, it is transported and modified in the liver before re-entering the circulation to be transported to other tissues, such as brain and muscles. Like other polyphenols, quercetin exerts anti-cancer activities, and has anti-oxidative and anti-inflammatory properties. Quercetin promotes nitric oxide production via the reduction of dietary nitrite in the stomach, and likely the circulation [34]. The strong reducing power of quercetin causes a reduction in the availability of nitrite that influences vascular function [34]. Quercetin has a negative effect on intracellular regulatory signaling events initiated by FceRI cross-linking and other activating receptors on mast cells [35]. Quercetin may exert physiological functions though the interaction with phosphatidylinositol-3-phosphate kinase (PI3K), mitogen-activated protein kinase (MAPK), extracellular signal regulated kinase (ERK), kinase (MEK) 1, and others [36]. Quercetin may also inhibit PI3K enzymatic activity by displacing ATP binding from PI3K and activate AMP-activated protein kinase (AMPK), which exert anticancer and antiinflammatory effects [37]. Therefore, besides possessing antioxidative properties, quercetin and its related polyphenols activate in the cells several biological functions which help organs, such as brain blood vessels, intestine and muscle, to work better [38]. Therefore, quercetin is an anti-oxidant polyphenol with potent anticar- 
cinogenic and anti-inflammatory activity. As an anti-inflammatory, quercetin has the capacity to influence arachidonic cascade products such as inhibition of prostaglandin E2 and LTB4 in acute inflammation induced by carrageenan in rodents [39]. Quercetin also exerts its anti-inflammatory properties by inhibiting mast cell degranulation and therefore all the pro-inflammatory compounds contained in granules, including tumor necrosis factor (TNF) [22].

Clinical studies indicate that quercetin and other flavonoids have cytoprotective activity, and when they are used together with other treatment modalities, flavonoids are useful for the treatment of acute and chronic inflammatory conditions such as asthma [40].

The major metabolite of quercetin is quercetin-3-O$\beta$-D-glucuronide, which exerts beneficial activity when distributed in the tissues and contributes to the activation of many physiological functions [37].

Quercetin has some effects in many organs including brain, blood vessels, muscle, intestine, liver, kidney, skin and bone, and influences neurodegenerative diseases, mood disorders, atherosclerosis, and metabolic syndrome, where mast cells are likely involved [37]. In addition, quercetin treatments of neurodegenerative diseases cause modulation of pro-inflammatory and anti-inflammatory cytokines and chemokines [41].

The flavonoid quercetin has a variety of functions including the inhibition of histamine release from human basophils and murine mast cells [42]. Moreover, quercetin is a potent anti-cytokine and chemokine generated from several cell types, which has an inhibitor effect in immunological and non-immunological conditions, mediated by mast cells [43]. It has been reported that quercetin inhibits, in a dose-response manner, tryptase and MCP-1 [27]. In addition, using RT-PCR, quercetin inhibits the transcription of histidine decarboxylase, the enzyme responsible for the generation of histamine from histidine. These data suggest that quercetin is a good candidate for reducing the release of pro-inflammatory mast cell mediators [27]. Quercetin inhibits contact dermatitis and photosensitivity in humans and suppresses ultraviolet irradiation-induced expression of inflammatory cytokines IL-1 $\beta$, IL-6, IL-8 and TNF in human keratinocytes [31].

Luteolin (3',4',5,7-tetrahydroxyflavone) is a flavone, a natural compound, with anti-oxidant properties and the capacity to inhibit proliferation of some cancer cells, with a mediator effect on mast cell-mediated allergy, and inflammatory and autoimmune disorders [44]. Luteolin is found in tea, olive fruit extract, vegetables and fruits, and is well tolerated and safe in children treated for autism spectrum disorders (ASD), demonstrating that it has neuroprotective activity [44]. Luteolin belongs to a flavone group of compounds (flavonoids), possesses anti-oxidant properties, inhibits proliferation of some cancer cells and exerts a regulatory effect on mast cell-mediated inflamma- tory diseases and allergy [45]. Moreover, luteolin inhibits T-cell activation, acute and chronic inflammation, and immune cells in patients affected by multiple sclerosis, reduces cognitive decline in rats and increases spatial memory in mice [46].

Recently, Theoharides et al. showed that luteolin treatment of activated human cultured keratinocytes in vitro inhibits their proliferation and suppresses TNF-induced production of inflammatory mediators IL-6, IL-8 and vascular endothelial growth factor (VEGF) [47]. In addition, they showed that luteolin decreases TNF-triggered activation of the transcription factor NF- $\kappa \mathrm{B}$ at both protein and gene transcription levels. These authors demonstrated that luteolin is a promising candidate for development into effective treatment for chronic inflammatory disorders.

Furthermore, other authors reported that luteolin has the capacity to arrest the cell cycle during the G1 phase in several forms of cancer cell proliferations in vivo and in vitro [48]. Luteolin is a strong activator of apoptosis with an unclear mechanism [49]. In several carcinomas, luteolin enhances the expression of Fas through triggering the degradation of signal transducer and activator of transcription 3 (STAT3) [50]. The biological activity of luteolin formula is due to the hydroxyl moieties and the 2-3 double bond. The intake of luteolin is inversely associated with subsequent coronary heart disease in some but not all prospective epidemiological studies [51].

The release of IL-1, IL-6 and TNF (inflammatory cytokines) after stimulation might lead to the recruitment of $\mathrm{T}$ cells and mast cells in the inflamed tissue and the generation of chemokines such as IL- 8 , an $\alpha$-chemokine (CXC chemokine), and/or MCP-1, a $\beta$-chemokine (CC chemokine) [26]. TNF has been shown to induce IL-8 (CXCL8) mRNA expression in a melanoma cell line and upregulate IL-8 receptor expression in normal melanocytes. IL-8 is a chemokine important in inflammatory skin diseases, and is produced by monocytes, mast cells, fibroblasts, endothelial cells, dendritic cells and keratinocytes. IL- 8 is chemotactic to neutrophils, T-lymphocytes, basophils and keratinocytes, and its gene expression is significantly increased in skin diseases, an effect inhibited by luteolin [52-56].

However, more studies on the therapeutic and neuroprotective effects of polyphenols are needed, and their precise mechanism of action still remains to be clarified.

This article was compiled from the data obtained in the laboratory of: Immunology Division, Medical School, University of Chieti, Chieti, Italy, and in the Molecular Immunopharmacology and Drug Discovery Laboratory, Medical School, Tufts University, Boston, USA. In addition, electronic literature searches were performed, and relevant articles were obtained and studied.

The authors declare no conflict of interest. 


\section{References}

1. Nahrstedt A, Schmidt M, Jäggi R, et al. (2007): Willow bark extract: the contribution of polyphenols to the overall effect. Wien Med Wochenschr 157: 348-351.

2. King L, Tsilioni I, Theoharides TC (2018): Time to look past TNF and thalidomide for cachexia - could mast cells and flavonoids be the answer? J Biol Regul Homeost Agents 32: 443-447.

3. Molitor C, Mauracher SG, Rompel A (2016): Aurone synthase is a catechol oxidase with hydroxylase activity and provides insights into the mechanism of plant polyphenol oxidases. Proc Natl Acad Sci U S A 113: E1806-1815.

4. Osawa T (1999): Protective role of dietary polyphenols in oxidative stress. Mech Ageing Dev 111: 133-139.

5. Liu CL, Yan L, Cai KR, et al. (2018): Effects of soybean isoflavones on $\mathrm{Wnt} / \beta$-catenin and the TGF- $\beta 1$ signaling pathway in renal tissue of type 2 diabetic rats. J Biol Regul Homeost Agents 32: 455-464.

6. Bahramsoltani R, Farzaei MH, Farahani MS, Rahimi R (2015): Phytochemical constituents as future antidepressants: a comprehensive review. Rev Neurosci 26: 699-719.

7. Yaniv SP, Lucki A, Klein E, Ben-Shachar D (2010): Dexamethasone enhances the norepinephrine-induced ERK/MAPK intracellular pathway possibly via dysregulation of the alpha2-adrenergic receptor: implications for antidepressant drug mechanism of action. Eur J Cell Biol 89: 712-722.

8. Li AN, Li S, Zhang YJ, et al. (2014): Resources and biological activities of natural polyphenols. Nutrients 6: 6020-6047.

9. Youdim KA, Joseph JA (2001): A possible emerging role of phytochemicals in improving age-related neurological dysfunctions: a multiplicity of effects. Free Radic Biol Med 30: 583-594.

10. Kawabata K, Mukai R, Ishisaka A (2015): Quercetin and related polyphenols: new insights and implications for their bioactivity and bioavailability. Food Funct 6: 1399-1417.

11. Gebicka L, Banasiak E (2009): Flavonoids as reductants of ferryl hemoglobin. Acta Biochim Pol 56: 509-513.

12. Albini A, Tosetti F, Li VW, et al. (2012): Cancer prevention by targeting angiogenesis. Nat Rev Clin Oncol 9: 498-509.

13. Yasueda A, Urushima H, Ito T (2016): Efficacy and interaction of antioxidant supplements as adjuvant therapy in cancer treatment: a systematic review. Integr Cancer Ther 15: 17-39.

14. Chen S (2011): Natural products triggering biological targets - a review of the anti-inflammatory phytochemicals targeting the arachidonic acid pathway in allergy asthma and rheumatoid arthritis. Curr Drug Targets 12: 288-301.

15. Cárdeno A, Sánchez-Hidalgo M, Aparicio-Soto M, et al. (2014): Extra virgin olive oil polyphenolic extracts downregulate inflammatory responses in LPS-activated murine peritoneal macrophages suppressing NFKB and MAPK signalling pathways. Food Funct 5: 1270-1277.

16. Bao L, Alexander JB, Shi VY, et al. (2014): Interleukin-4 up-regulation of epidermal interleukin-19 expression in keratinocytes involves the binding of signal transducer and activator of transcription 6 (Stat6) to the imperfect Stat6 sites. Immunology 143: 601-608.

17. Granato T, Anastasi E, Viggiani V, et al. (2018): Serum 25-hydroxy vitamin D levels in essential hypertension. J Biol Regul Homeost Agents 32: 1599-1604.

18. Jasinska T, Wyszyska-Chlap M, Kasperski J, Kasperska-Zajac A (2015): Plasma soluble CD40 concentration in patients with delayed pressure urticaria. Eur J Inflamm 13: 126-129.
19. Hussain F, Irshad A, Shahid M (2018): Study of selected therapeutic potentials of Momordica charantia fruit. J Biol Regul Homeost Agents 32: 859-862.

20. Pałgan K, Bartuzi Z (2015): Platelet activating factor in allergies. Int J Immunopathol Pharmacol 28: 584-589.

21. Cottrell GS, Coelho AM, Bunnett NW (2002): Protease-activated receptors: the role of cell-surface proteolysis in signalling. Essays Biochem 38: 169-183.

22. Floreani AA, Rennard SI (1997): Experimental treatments for asthma. Curr Opin Pulm Med 3: 30-41.

23. Theoharides TC, Enakuaa S, Sismanopoulos N, et al. (2012): Contribution of stress to asthma worsening through mast cell activation. Ann Allergy Asthma Immunol 109: 14-19.

24. Kurvers HA (1998): Reflex sympathetic dystrophy: facts and hypotheses. Vasc Med 3: 207-214.

25. Liu HW, Meng Y, Zhang J, et al. (2018): Role and influence of p75NTR receptor on antioxidative damage of retinal pigment epithelial cells. J Biol Regul Homeost Agents 32: 13791387.

26. Guan D, Li Y, Peng X, et al. (2018): Thymoquinone protects against cerebral small vessel disease: Role of antioxidant and anti-inflammatory activities. J Biol Regul Homeost Agents 32: 225-231.

27. Kempuraj D, Castellani ML, Petrarca C, et al. (2006): Inhibitory effect of quercetin on tryptase and interleukin-6 release, and histidine decarboxylase mRNA transcription by human mast cell-1 cell line. Clin Exp Med 6: 150-156.

28. Conti P, Pang X, Boucher W, et al. (1997): Impact of Rantes and MCP-1 chemokines on in vivo basophilic cell recruitment in rat skin injection model and their role in modifying the protein and mRNA levels for histidine decarboxylase. Blood 89: 4120-4127.

29. Kletke O, Sergeeva OA, Lorenz P, et al. (2013): New insights in endogenous modulation of ligand-gated ion channels: histamine is an inverse agonist at strychnine sensitive glycine receptors. Eur J Pharmacol 710: 59-66.

30. Beghdadi W, Porcherie A, Schneider BS, et al. (2008): Inhibition of histamine-mediated signaling confers significant protection against severe malaria in mouse models of disease. J Exp Med 205: 395-408.

31. Weng Z, Zhang B, Asadi S, et al. (2012): Quercetin is more effective than cromolyn in blocking human mast cell cytokine release and inhibits contact dermatitis and photosensitivity in humans. PLoS One 7: e33805.

32. Plemel JR, Juzwik CA, Benson CA, et al. (2015): Over-thecounter anti-oxidant therapies for use in multiple sclerosis: A systematic review. Mult Scler 21: 1485-1495.

33. Russo M, Spagnuolo C, Tedesco I, et al. (2012): The flavonoid quercetin in disease prevention and therapy: facts and fancies. Biochem Pharmacol 83: 6-15.

34. Olszanecki R, Gebska A, Kozlovski VI, Gryglewski RJ (2002): Flavonoids and nitric oxide synthase. J Physiol Pharmacol 53 (4 Pt 1): 571-584.

35. Kandere-Grzybowska K, Kempuraj D, Cao J, et al. (2006): Regulation of IL-1-induced selective IL-6 release from human mast cells and inhibition by quercetin. Br J Pharmacol 148: 208-215.

36. Kyaw M, Yoshizumi M, Tsuchiya K, et al. (2004): Atheroprotective effects of antioxidants through inhibition of mitogen-activated protein kinases. Acta Pharmacol Sin 25: 977985 . 
37. Nabavi SF, Russo GL, Daglia M, Nabavi SM (2015): Role of quercetin as an alternative for obesity treatment: you are what you eat! Food Chem 179: 305-310.

38. Kawabata K, Mukai R, Ishisaka A (2015): Quercetin and related polyphenols: new insights and implications for their bioactivity and bioavailability. Food Funct 6: 1399-1417.

39. Welton AF, Hurley J, Will P (1988): Flavonoids and arachidonic acid metabolism. Prog Clin Biol Res 280: 301-312.

40. Nam NH (2006): Naturally occurring NF-kappaB inhibitors. Mini Rev Med Chem 6: 945-951.

41. Jeong E, Lee JY (2011): Intrinsic and extrinsic regulation of innate immune receptors. Yonsei Med J 52: 379-392.

42. Middleton E Jr. (1986): Effect of flavonoids on basophil histamine release and other secretory systems. Prog Clin Biol Res 213: 493-506.

43. Peluso I, Miglio C, Morabito G, et al. (2015): Flavonoids and immune function in human: a systematic review. Crit Rev Food Sci Nutr 55: 383-395.

44. Theoharides TC, Conti P, Economu M (2014): Brain inflammation, neuropsychiatric disorders, and immunoendocrine effects of luteolin. J Clin Psychopharmacol 34: 187-189.

45. Lin Y, Shi R, Wang X, Shen HM (2008): Luteolin, a flavonoid with potential for cancer prevention and therapy. Curr Cancer Drug Targets 8: 634-646.

46. Kempuraj D, Tagen M, Iliopoulou BP, et al. (2008): Luteolin inhibits myelin basic protein-induced human mast cell activation and mast cell-dependent stimulation of Jurkat $\mathrm{T}$ cells. Br J Pharmacol 155: 1076-1084.

47. Weng Z, Patel AB, Vasiadi M, et al. (2014): Luteolin inhibits human keratinocyte activation and decreases NF- $\mathrm{KB}$ induction that is increased in psoriatic skin. PLoS One 9: e90739.

48. Aneknan P, Kukongviriyapan V, Prawan A, et al. (2014): Luteolin arrests cell cycling, induces apoptosis and inhibits the JAK/STAT3 pathway in human cholangiocarcinoma cells. Asian Pac J Cancer Prev 15: 5071-5076.

49. George VC, Naveen Kumar DR, Suresh PK, et al. (2013): Comparative studies to evaluate relative in vitro potency of luteolin in inducing cell cycle arrest and apoptosis in $\mathrm{HaCaT}$ and A375 cells. Asian Pac J Cancer Prev 14: 631-637.

50. Zhang H, Li X, Zhang Y, Luan X (2014): Luteolin induces apoptosis by activating Fas signaling pathway at the receptor level in laryngeal squamous cell line Hep-2 cells. Eur Arch Otorhinolaryngol 271: 1653-1659.

51. Hollman PC, Katan MB (1999): Health effects and bioavailability of dietary flavonols. Free Radic Res 31 Suppl: S75-80.

52. Miniati A, Weng Z, Zhang B, et al. (2014): Stimulated human melanocytes express and release interleukin-8, which is inhibited by luteolin: relevance to early vitiligo. Clin Exp Dermatol 39: 54-57.

53. Kritas SK, Gallenga CE, D Ovidio C, et al. (2018): Impact of mold on mast cell-cytokine immune response. J Biol Regul Homeost Agents 32: 763-768.

54. Aragona SE, Grassi FR, Nardi G, et al. (2017): Photobiomodulation with polarized light in the treatment of cutaneous and mucosal ulcerative lesions. J Biol Regul Homeost Agents 31 (2 Suppl. 2): 213-218.

55. Nisticň S, Torchia V, Gliozzi M, et al. (2016): Pharmacoeconomy of drugs used in the treatment of actinic keratoses. Int J Immunopathol Pharmacol 29: 796-804.

56. Park SH, Choi H-J, Lee S, Han J-S (2015): TLR4-mediated IRAK1 activation induces TNF- $\alpha$ expression via JNK-dependent NF- $\kappa$ B activation in human bronchial epithelial cells. Eur J Inflamm 13: 183-195. 